\title{
Brain glycogen: emergency fuel and dynamic function in neurotransmission
}

\author{
Anne B. Walls • Arne Schousboe
}

Received: 2 September 2014 / Accepted: 19 September 2014 /Published online: 28 September 2014

(C) Springer Science+Business Media New York 2014

The outdated view that brain glycogen serves as a latent pool of glucose units awaiting a devastating abruption in the continuous glucose supply was fundamentally changed by the observation that glycogen is degraded under physiological conditions in response to neuronal activation. This finding attracted neuroscientists' attention to investigate the biochemical aspects of glycogen in brain metabolism. This interest was further strengthened by the discovery that glycogen plays a critical role in higher cognitive functions, including learning and memory. Throughout the last decade, some major steps have been taken in settling the functional and metabolic roles of glycogen in the brain. It has been learned that brain glycogen is extremely dynamic, as it features continuous shunting of glucose units into and out of its outer layers. Moreover, studies in chickens revealed that brain glycogen is important not only as an energy substrate but also as a precursor for glutamate and glutamine biosynthesis, suggesting a role for glycogen in maintenance of neurotransmitter homeostasis. On this basis we found it relevant and timely to assemble different aspects of the current understanding of brain glycogen in a special issue of Metabolic Brain Disease. This issue encompasses articles depicting the role of glycogen as an energy substrate for astrocytes and neurons in both the central and peripheral nervous system. Although the use of glycogen as an energy source has been established for quite some time, the understanding of the specific cellular mechanisms fueled by its degradation is being continuously elaborated and provides a new perspective to re-evaluate previous findings. Other articles discuss novel aspects of glycogen metabolism in diseases, such as diabetes, Alzheimer's disease and epilepsy, suggesting the involvement of abnormal glycogen metabolism in pathological conditions. Altogether, these articles cover the current understanding of the functional and metabolic aspects of brain glycogen and portray its multifaceted role for the maintenance of proper brain function.

\footnotetext{
A. B. Walls $\cdot$ A. Schousboe $(\square)$

Department of Drug Design and Pharmacology, Faculty of Health and Medical Sciences, University of Copenhagen, Copenhagen, Denmark

e-mail: arne.schousboe@sund.ku.dk
}

\author{
A. B. Walls \\ e-mail: abw@sund.ku.dk
}

